\title{
Valenced action/inhibition learning in humans is modulated by a genetic variant linked to dopamine $\mathrm{D} 2$ receptor expression
}

\author{
Anni Richter ${ }^{1}$, Marc Guitart-Masip ${ }^{2,3}$, Adriana Barman ${ }^{1}$, Catherine Libeau ${ }^{1}$, Gusalija Behnisch ${ }^{1}$, \\ Sophia Czerney ${ }^{1}$, Denny Schanze ${ }^{4}$, Anne Assmann ${ }^{1}$, Marieke Klein ${ }^{1+}$, Emrah Düzel ${ }^{5,6,78}$, \\ Martin Zenker ${ }^{4}$, Constanze I. Seidenbecher ${ }^{1,8}$ and Björn H. Schott ${ }^{1,8,9,10 *}$
}

\author{
1 Department of Neurochemistry and Molecular Biology, Department of Behavioral Neurology, Leibniz Institute for Neurobiology, Magdeburg, Germany \\ ${ }^{2}$ Wellcome Trust Centre for Neuroimaging, Institute of Neurology, University College London, London, UK \\ ${ }^{3}$ Ageing Research Centre, Karolinska Institute, Stockholm, Sweden \\ ${ }^{4}$ Institute of Human Genetics, Otto von Guericke University of Magdeburg, Magdeburg, Germany \\ ${ }^{5}$ Institute of Cognitive Neurology and Dementia Research, Otto von Guericke University Magdeburg, Magdeburg, Germany \\ ${ }^{6}$ Institute of Cognitive Neuroscience, University College London, London, UK \\ ${ }^{7}$ German Center for Neurodegenerative Diseases, Magdeburg, Germany \\ ${ }^{8}$ Center for Behavioral Brain Sciences, Otto von Guericke University of Magdeburg, Magdeburg, Germany \\ ${ }^{9}$ Department of Psychiatry, Charité University Hospital, Berlin, Germany \\ ${ }^{10}$ Department of Neurology, University of Magdeburg, Magdeburg, Germany
}

Edited by:

Daniela Laricchiuta, IRCCS Santa

Lucia Foundation, Italy

Reviewed by:

C. Nico Boehler, Ghent University, Belgium

Alexander Strobel, Technische

Universitaet Dresden, Germany

*Correspondence:

Björn H. Schott, Leibniz Institute for

Neurobiology, Brenneckestr. 6,

39118 Magdeburg, Germany

e-mail: bschott@neuro2.

med.uni-magdeburg.de

${ }^{\dagger}$ Present address:

Marieke Klein, Department of

Human Genetics, Donders Institute

for Brain, Cognition and Behaviour,

Radboud University Medical Centre,

Nijmegen, Netherlands
Motivational salience plays an important role in shaping human behavior, but recent studies demonstrate that human performance is not uniformly improved by motivation. Instead, action has been shown to dominate valence in motivated tasks, and it is particularly difficult for humans to learn the inhibition of an action to obtain a reward, but the neural mechanism behind this behavioral specificity is yet unclear. In all mammals, including humans, the monoamine neurotransmitter dopamine is particularly important in the neural manifestation of appetitively motivated behavior, and the human dopamine system is subject to considerable genetic variability. The well-studied TaqIA restriction fragment length polymorphism (rs1800497) has previously been shown to affect striatal dopamine metabolism. In this study we investigated a potential effect of this genetic variation on motivated action/inhibition learning. Two independent cohorts consisting of 87 and 95 healthy participants, respectively, were tested using the previously described valenced go/no-go learning paradigm in which participants learned the reward-associated no-go condition significantly worse than all other conditions. This effect was modulated by the TaqlA polymorphism, with carriers of the A1 allele showing a diminished learning-related performance enhancement in the rewarded no-go condition compared to the A2 homozygotes. This result highlights a modulatory role for genetic variability of the dopaminergic system in individual learning differences of action-valence interaction.

Keywords: dopamine D2 receptor, TaqIA, reward learning, motivated learning, action bias

\section{INTRODUCTION}

Efficient decision making requires an individual to select responses that maximize reward and minimize punishment or loss. Such motivated behavior involves two fundamental axes of control, namely valence-spanning reward and punishment, and action-spanning invigoration and inhibition. Previous studies have shown that these two axes are not independent (GuitartMasip et al., 2012b, 2013; Cavanagh et al., 2013; Chowdhury et al., 2013; for review see Guitart-Masip et al., 2014) and that decision making is not only influenced by an instrumental controller that learns to optimize choices on the basis of their contingent consequences, but also on a Pavlovian controller that generates stereotyped, "hard-wired" behavioral responses to the occurrence of motivationally salient outcomes or learned predictions of such outcomes (Dickinson and Balleine, 2002; Guitart-Masip et al., 2013). The presence of such "hard-wired" response patterns may be an evolutionarily beneficial adaptation to an environment world in which obtaining a reward typically requires some sort of overt behavioral response (go to win) whereas avoiding a punishment rather requires an avoidance of those actions that may lead to it (no-go to avoid losing). On the other hand, such a response bias may also be a source of suboptimal behavior when Pavlovian and instrumental controllers are in opposition (Breland and Breland, 1961; Dayan et al., 2006; Boureau and Dayan, 2011).

In order to manipulate action and valence orthogonally, Guitart-Masip et al. (2012b) designed a go/no-go learning task that involves besides the commonly investigated conditions go to win and no-go to avoid losing also the vice versa conditions where 
the participant needs to perform an action to avoid a punishment (go to avoid losing) or to inhibit an action to obtain a reward (nogo to win). Studies employing this task have repeatedly shown that while active choices in rewarded conditions and passive choices in punished conditions can be learned easily, it is significantly harder to learn an approach behavior to avoid a punishment and yet even more difficult to inhibit an action to obtain a reward. This asymmetry indicates that signals that predict reward are prepotently associated with behavioral activation, whereas signals that predict punishment are intrinsically coupled to behavioral inhibition.

In search for neural mechanisms underlying this behavioral asymmetry in the coupling between action and valence, monoaminergic, particularly dopaminergic, neuromodulation is a prime candidate (Gray and McNaughton, 2000; Boureau and Dayan, 2011; Cools et al., 2011). Dopamine (DA) is believed to enable or enhance the generation of active motivated behavior (Berridge and Robinson, 1998; Niv et al., 2007; Salamone et al., 2007; Beierholm et al., 2013) and to support instrumental learning (Frank et al., 2004; Daw and Doya, 2006; Wickens et al., 2007). It has been observed that DA depletion leads to decreased motor activity and decreased motivated behavior (Ungerstedt, 1971; Palmiter, 2008), along with decreased vigor or motivation to work for rewards in demanding reinforcement schedules (Salamone et al., 2005; Niv et al., 2007). Conversely, boosting DA levels with levodopa invigorates motor responses in healthy humans (Guitart-Masip et al., 2012a) and DA promotes "go" and impairs "no-go" learning, for example in patients with Parkinson's disease (Frank et al., 2004). However, contrary to the expectations suggested by this evidence, administration of levodopa reduced the learning disadvantage of the no-go to win condition when compared to the no-go to avoid losing (Guitart-Masip et al., 2013). These effects suggested that DA is involved in decreasing the coupling between action and valence, supposedly via DA's actions on neural functions implemented in prefrontal cortex (Hitchcott et al., 2007). It is therefore unclear how striatal DA modulates the coupling between action and valence uncovered in this task.

The aim of the present study was to test whether naturally occurring differences in healthy humans in this valenced action/inhibition learning might arise from dopaminergic mechanisms and how striatal DA effects the action/valence interaction. To address this issue, we used the valenced go/no-go learning paradigm in a cohort of young, healthy subjects, and tested them for the TaqIA restriction length polymorphism (rs1800497), a common genetic variation of the dopamine D2 receptor (DRD2) gene known to affect D2 receptor expression and striatal DA metabolism. Although the underlying molecular mechanisms are yet not fully understood, the TaqIA polymorphism has been repeatedly associated with reduced striatal DRD2 density in A1 carriers as evident from three post mortem studies (Noble et al., 1991; Thompson et al., 1997; Ritchie and Noble, 2003) and two out of three conducted in vivo binding studies (Laruelle et al., 1998; Pohjalainen et al., 1998; Jonsson et al., 1999). Laakso et al. (2005) suggested that the lower D2 receptor expression leads to decreased autoreceptor function, thereby increasing the DA and/or trace amine synthesis rate in the brains of A1 allele carriers. Moreover, Kirsch et al. (2006) observed an increase of striatal BOLD signal in response to the dopamine D2 receptor agonist bromocriptine in subjects carrying the A1 allele, but not in subjects without the A1 allele, and Stelzel et al. (2010) reported a generally increased striatal BOLD signal in A1 carriers. As striatal BOLD signal has been shown to correlate with DA release (Schott et al., 2008), the increased striatal activation in A1 carriers might be related to higher presynaptic dopaminergic activity (Richter et al., 2013). Because striatal DA is associated with linking action with reward (Berridge and Robinson, 1998; Frank et al., 2004; Daw and Doya, 2006; Niv et al., 2007; Salamone et al., 2007; Wickens et al., 2007; Beierholm et al., 2013), we hypothesized that A1 carriers might show increased coupling between action and valence.

\section{MATERIALS AND METHODS PARTICIPANTS}

Participants were recruited from a cohort of 719 young healthy volunteers of Caucasian ethnicity of a large-scale behavioral genetic study conducted at the Leibniz Institute for Neurobiology, Magdeburg. Given our hypothesis regarding differential performance in the valenced go/no-go task as a function of striatal D2 receptor availability, we selected participants a priori as a function of DRD2 TaqIA genotype. To control for confounding effects of genetic influences on prefrontal DA availability, we also ensured a balanced distribution of the COMT Val108/158 Met polymorphism that is known to affect prefrontal DA levels and D1 receptor binding (Gogos et al., 1998; Matsumoto et al., 2003; Meyer-Lindenberg et al., 2005; Slifstein et al., 2008). All participants were right-handed according to self-report, not genetically related, and had obtained at least a university entrance diploma (Abitur) as educational certificate. Importantly, all participants had undergone routine clinical interview to exclude present or past neurological or psychiatric illness, alcohol, or drug abuse, use of centrally acting medication, the presence of psychosis or bipolar disorder in a first-degree relative, and additionally, given the design of the experiment, regular gambling. Two independent cohorts of healthy participants were tested (cohort 1: 43 females and 44 males; age: range 19-36 years, mean 24.6 years, $S D=3.1$ years; cohort 2: 48 females and 47 males; age: range $20-33$ years, mean 24.6 years, $S D=2.8$ years). Because of a previously reported potential association of the A1 allele with nicotine consumption (Verde et al., 2011; for reviews see Comings and Blum, 2000; Lerman et al., 2007), smoking status was assessed from the participants. All participants gave written informed consent in accordance with the Declaration of Helsinki and received financial compensation for participation. The work was approved by the Ethics Committee of the University of Magdeburg, Faculty of Medicine.

\section{GENOTYPING}

The DRD2/ANKK1 TaqIA restriction length polymorphism (NCBI accession number: rs1800497) was genotyped using a protocol previously described in Richter et al. (2013). Genomic DNA was extracted from blood leukocytes using the GeneMole ${ }^{\circledR}$ automated system (Mole Genetics AS, Lysaker, Norway) according to the manufacturer's protocol. Genotyping was performed using PCR followed by allele-specific restriction analysis using previously described primers (Grandy et al., 1989). Genotyping was 
also performed for several additional polymorphisms, including COMT Val108/158 Met (see Table 1), to control for confounding effects of other genetic variants and to reduce the risk of population stratification.

\section{PARADIGM}

We used a previously employed go/no-go learning task with orthogonalized action requirements and outcome valence (Guitart-Masip et al., 2012b, 2013; Chowdhury et al., 2013). The trial timing is displayed in Figure 1. Each trial consisted of presentation of a fractal cue, a target detection task, and a probabilistic outcome. First, one out of four abstract fractal cues was displayed for $1000 \mathrm{~ms}$. Participants were informed that a fractal indicated whether they would subsequently be required to perform a target detection task by pressing a button (go) or not (no-go) and that the cue also indicated the possible valence of the outcome of the subjects' behavior (reward/no reward or punishment/no punishment). However, subjects were not instructed about the contingencies for each fractal image and had to learn them by trial and error. The meaning of the fractal images was randomized across participants. Following a variable interval $(250-3500 \mathrm{~ms})$ after offset of the fractal image, the target detection task started: participants had the opportunity to press a button within a time limit of $2000 \mathrm{~ms}$ to indicate the side of a circle for go trials, or not to press for no-go trials. After the offset of the circle after 1500 and $1000 \mathrm{~ms}$ of fixation, subjects were presented with the outcome. The outcome remained on screen for $2000 \mathrm{~ms}$ and after a variable intertrial interval (ITI; 750-1500 ms) a new trial started. Participants were informed that the outcome was probabilistic: in win trials $80 \%$ of correct choices and $20 \%$ of incorrect choices were rewarded with $0.50 €$ (the remaining $20 \%$ of correct and $80 \%$ of incorrect choices leading to no outcome), while in avoid losing trials $80 \%$ of correct choices and $20 \%$ of incorrect choices avoided a loss of $0.50 €$ (the remaining $20 \%$ of correct and $80 \%$ of incorrect choices leading to a punishment). Thus, there were four trial types depending on the nature of the fractal cue presented at the beginning of the trial: press the correct button in the target detection task to gain a reward ( $g$ o to win); press the correct button in the target detection task to avoid punishment (go to avoid losing); do not press a button in the target detection task to gain a reward (no-go to win); do not press a button in the target detection task to avoid punishment (no-go to avoid losing). The task included 240 trials, 60 trials per condition and was divided into four sessions $9 \mathrm{~min}$ each ( 15 trials per condition in randomized order). Subjects were told that they would be paid their earnings of the task up to a total of $25 €$ and a minimum of $7 €$. Before starting with the learning task, subjects performed 10 trials of the target detection task in order to get familiarized with the speed requirements.

\section{STATISTICAL ANALYSIS}

The percentage of correct choices in the target detection task (correct button press for go conditions and correct omission of responses in no-go trials) was collapsed across time bins of 30 trials per condition and analyzed with a mixed ANOVA with time (1st/2nd half), action (go/no-go), and valence (win/lose) as within-subject factors and TaqIA genotype $(\mathrm{A} 1+/ \mathrm{A} 1-)$ as between-subject factor. Additionally reaction times of correct

Table 1 | Genotyped polymorphisms.

\begin{tabular}{|c|c|c|}
\hline Polymorphism/Gene & NCBI accession number & Genotyping protocol \\
\hline DAT1 VNTR & rs28363170 & $\begin{array}{l}\text { Schott et al., } 2006 \\
\text { Primers for PCR: } \\
\text { 5'-TGTGGTGTAGGAAACGGCCTGAG-3' } \\
\text { 5'-CTTCCTGGAGGTCACGGCTCAAAGG-3' } \\
\text { PCR products were not digested }\end{array}$ \\
\hline DRD2 C957T & rs6277 & $\begin{array}{l}\text { Kompetitive allele-specific PCR (KASP) } \\
\text { Assay on Demand (LGC Genomics, Berlin, } \\
\text { Germany) }\end{array}$ \\
\hline DARPP-32 & rs907094 & $\begin{array}{l}\text { Primers for PCR: } \\
\text { 5'-GCACCCCATGGAGCGAGAAGACAG-3' } \\
\text { 5'-CGCATTGCTGAGTCTCACCTGCAGTC-3' } \\
\text { Restriction enzyme: Tru1। }\end{array}$ \\
\hline
\end{tabular}




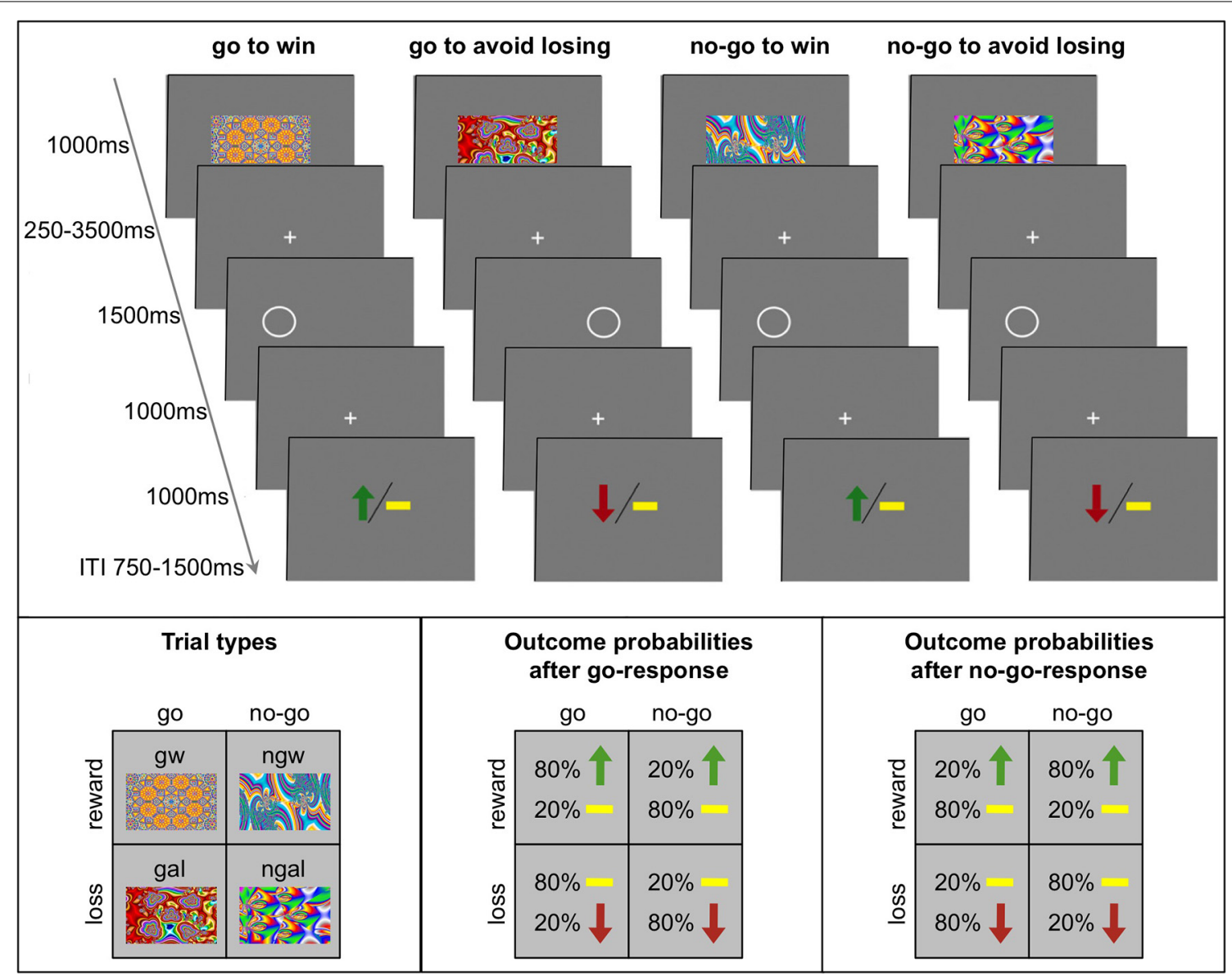

FIGURE 1 | Experimental paradigm of the probabilistic monetary go/no-go task. Fractal images indicate the combination between action (go or no-go) and valence (reward or loss). On go trials, subjects press a button for the side of a circle. On no-go trials they withhold a response. Arrows indicate rewards (green) or losses (red). Horizontal bars (yellow) symbolize the absence of a win or a loss.
The schematics at the bottom represent for each trial type the nomenclature (left), the possible outcomes and their probabilities after response to the target ("go"; middle), and the possible outcomes and their probability after withholding a response to the target ("no-go"; right). gw, go to win; gal, go to avoid losing; ngw, no-go to win; ngal, no-go to avoid losing; ITI, intertrial interval. go responses (RTs) were analyzed using a mixed ANOVA with valence (win/lose) and TaqIA genotype $(\mathrm{A} 1+/ \mathrm{A} 1-)$ as factors. When appropriate, paired $t$-test, independent sample $t$-test or Mann-Whitney $U$-test were used as post-hoc tests.

The analysis of the behavioral data was done in two stages. In cohort 1 we included the TaqIA and the COMT Val108/158 Met polymorphism as between-subject factors. In the second we specifically aimed to replicate the significant effect of TaqIA. The following statistics include TaqIA as the only between-subject factor.

\section{RESULTS \\ GENOTYPING}

Genotyping was performed in the entire cohort of 719 subjects, and two sub-cohorts were recruited based on the DRD2/ANKK1 TaqIA genotype. The data of 87 participants in cohort 1 and 95 participants in cohort 2 were analyzed. In cohort 1 , we identified 4 A1 homozygotes, 33 heterozygotes and 50 A2 homozygotes. In cohort 2, genotyping revealed $4 \mathrm{~A} 1$ homozygotes, 30 heterozygotes and 61 A2 homozygotes. The distributions in both groups were at Hardy-Weinberg equilibrium (cohort $1: \chi^{2}=0.24$, $p=0.621$; cohort $\left.2: \chi^{2}=0.02, p=0.898\right)$. Al carriers $(\mathrm{A} 1+$ : $\mathrm{A} 1 / \mathrm{A} 1$ and A1/A2) were grouped together for all subsequent analyses as in previous behavioral and imaging studies of the TaqIA polymorphism (Stelzel et al., 2010; Richter et al., 2013). The groups $\mathrm{A} 1+$ and $\mathrm{A} 1-(\mathrm{A} 2 / \mathrm{A} 2)$ did not differ in gender, in age or in the number of smokers and nonsmokers (Table 2).

To control for effects of prefrontal DA availability, participants were also selected regarding the COMT Val108/158 Met (NCBI accession number: rs4680) polymorphism. Genotyping revealed $31 \mathrm{Met} / \mathrm{Met}, 29 \mathrm{Val} / \mathrm{Met}$, and $27 \mathrm{Val} / \mathrm{Val}$ carriers in cohort 1 and $30 \mathrm{Met} / \mathrm{Met}, 41 \mathrm{Val} / \mathrm{Met}$, and $24 \mathrm{Val} / \mathrm{Val}$ carriers in cohort 2. Allelic distribution for the COMT Val108/158 Met polymorphism did not differ significantly for either TaqIA A1 carriers or A2 homozygotes (Table 2). The experimenters who performed the behavioral task were blinded regarding DRD2/ANKK1 and COMT genotypes.

To further control for effects of population stratification and potential effects of putatively functional genetic variations in the dopamine system, genotyping was also performed for the 


\begin{tabular}{lccc}
\hline & A1+ & A1- & \\
\hline COHORT 1 & & $26 / 24$ & $\chi^{2}=0.31, p=0.577$ \\
Women/Men $(n=87)$ & $17 / 20$ & $24.3 \pm 2.6$ & $t_{(85)}=0.83, p=0.410$ \\
Mean age $(n=87)$ & $24.9 \pm 3.6$ & $14 / 36$ & $\chi^{2}=1.51, p=0.220$ \\
Smokers/Nonsmokers $(n=87)$ & $15 / 22$ & $18 / 15 / 17$ & $\chi^{2}=0.73, p=0.694$ \\
COMT mm/vm/vv $(n=87)$ & $13 / 14 / 10$ & $15 / 34$ & $\chi^{2}<0.01, p=0.996$ \\
DAT1-VNTR 9+/9- $(n=85)$ & $11 / 25$ & $8 / 24 / 18$ & $\chi^{2}=4.04, p=0.132$ \\
C957T CC/CT/TT $(n=87)$ & $11 / 19 / 7$ & $29 / 19 / 2$ & $\chi^{2}=0.19, p=0.912$ \\
DARPP-32 CC/CT/TT $(n=87)$ & $20 / 15 / 2$ & & \\
COHORT 2 & & & $\chi^{2}=3.20, p=0.074$ \\
Women/Men $(n=95)$ & $13 / 21$ & $24.2 \pm 2.4$ & $t_{(93)}=1.58, p=0.121$ \\
Mean age $(n=95)$ & $25.2 \pm 3.3$ & $14 / 47$ & $\chi^{2}=0.93, p=0.335$ \\
Smokers/Nonsmokers $(n=95)$ & $5 / 29$ & $19 / 27 / 15$ & $\chi^{2}=0.09, p=0.957$ \\
COMT mm/vm/vv $(n=95)$ & $11 / 14 / 9$ & $32 / 27$ & $\chi^{2}=0.16, p=0.693$ \\
DAT1-VNTR 9+/9- $(n=93)$ & $17 / 17$ & $3 / 37 / 21$ & $\chi^{2}=25.49, p<0.001$ \\
C957T CC/CT/TT $(n=95)$ & $15 / 17 / 2$ & $41 / 20 / 0$ & $\chi^{2}=0.8 .53, p=0.014$ \\
DARPP-32 CC/CT/TT $(n=95)$ & $15 / 16 / 3$ & & \\
\hline
\end{tabular}

Gender distribution, age (means \pm standard deviations), number of smokers and nonsmokers. Allelic distributions for following polymorphisms: COMT Val108/158 Met (mm, met homozygotes; vm, val/met heterozygotes; mm, met homozygotes), DAT1-VNTR (9+, carriers of the 9-repeat allele 9/9 and 9/10; 9-, 10-repeat homozygous subjects 10/10), C957T (CC/CT/TT carriers) and DARPP-32 (CC/CT/TT carriers). A1+; carriers of the A1 allele. A1-; A2 homozygotes.

DAT1-VNTR (NCBI accession number: rs28363170), the C957T polymorphism within the DRD2 gene (NCBI accession number: rs6277) and the DARPP-32 polymorphism (NCBI accession number: rs907094) (see Table 1). Allelic distributions for the DAT1-VNTR polymorphism did not differ significantly for either TaqIA A1 carriers or A2 homozygotes (Table 2). However, because of differences for the C957T and the DARPP-32 polymorphism, we additionally calculated an ANCOVA including these two polymorphisms as covariates (see below).

\section{BEHAVIORAL RESULTS}

We initially performed an omnibus mixed-design ANOVA to test for effects of both DRD2/ANKK1 and COMT genotypes. There was a significant four-fold interaction of DRD2/ANKK1 TaqIA with action, time and valence $\left[F_{(1,81)}=5.11, p=0.027\right]$, but no effect of COMT Val108/158 Met polymorphism (all $p>0.120$ ). All further analyses were therefore focused on the DRD2/ANKK1 TaqIA polymorphism. We computed as ANOVA for repeated measures on the percentage of correct (optimal) choices with action (go/no-go), valence (win/lose) and time (1st/2nd half) as within-subject factors and genotype $(\mathrm{A} 1+/ \mathrm{A} 1-)$ as betweensubject factor. See Table 3 for statistics.

Our study reproduced a main effect of action [cohort 1: $F_{(1,85)}=62.56, p<0.001 ;$ cohort $\left.2: F_{(1,93)}=50.87, p<0.001\right]$ and an action by valence interaction [cohort $1: F_{(1,85)}=44.41$, $p<0.001$; cohort 2 : $\left.F_{(1,93)}=37.72, p<0.001\right]$, as demonstrated in previous studies (Guitart-Masip et al., 2012b, 2013; Cavanagh et al., 2013; Chowdhury et al., 2013). Subjects showed better performance in conditions requiring a go choice than in trials requiring a no-go choice [cohort 1: $t_{(86)}=7.97, p<0.001$; cohort 2: $\left.t_{(94)}=7.68, p<0.001\right]$, and while they were better at learning from reward as compared to punishment in the go condition [cohort 1: $t_{(86)}=6.28, p<0.001$; cohort 2 : $t_{(94)}=$
5.74, $p<0.001]$, this relation reversed in the no-go condition [cohort 1: $t_{(86)}=4.99, p<0.001$; cohort $2: t_{(94)}=4.63, p<$ 0.001]. As Guitart-Masip et al. (2012b, 2013) we also observed a main effect of time [cohort 1: $F_{(1,85)}=135.92, p<0.001$; cohort 2: $\left.F_{(1,93)}=189.21, p=<0.001\right]$ and additionally an action by time interaction [cohort 1: $F_{(1,85)}=19.09, p<0.001$; cohort 2: $\left.F_{(1,93)}=59.77, p<0.001\right]$, indicating a preponderant initial bias toward go responses [cohort 1: $t_{(86)}=4.62, p<0.001$; cohort 2: $\left.t_{(94)}=8.46, p<0.001\right]$.

Most interestingly for the current study, we observed a fourfold interaction of action by valence by time by genotype [cohort 1: $F_{(1,85)}=5.24, p=0.025$; cohort $2: F_{(1,93)}=4.59$, $p=0.035]$. This effect was observed in the absence of an action by valence by genotype effect (cohort $1: p=0.811$; cohort 2 : $p=0.087$ ). While the genotype groups did not differ significantly in their mean performance in the first and second time bin in any condition (cohort $1: p>0.143$; cohort $2: p>0.167$ ), they showed a different degree of improvement from the first to the second time interval (learning gain: mean performance 2nd half-mean performance 1st half; see Figure 2). Performance of the A2 homozygotes in the no-go to win condition showed increased improvement from the first to the second half of the experiment compared to the A1 carriers [cohort 1: $t_{(85)}=2.78$, $p=0.007$ ]. In the second cohort this result was replicated [cohort $2: t_{(93)}=2.16, p=0.033$ ] , and A1 carriers showed lower performance in the go to avoid losing condition [cohort 2: $t_{(93)}=2.26$, $p=0.026]$. Because performance in the no-go to win condition during early trials differed between the two cohorts, we tested whether the observed interaction, which would likely reflect a difference in learning rate, remained significant when combining both datasets. A Three-Way ANCOVA across both cohorts (including cohort as a covariate of no interest; see Figure 2) revealed the same three-way interaction revealed by the analyses 
Table 3 | Statistics on percentage of correct responses.

\begin{tabular}{|c|c|c|}
\hline Effects & Cohort 1 & Cohort 2 \\
\hline Action & $\begin{array}{l}F_{(1,85)}=62.56 \\
p<0.001, \eta^{2}=0.42\end{array}$ & $\begin{array}{l}F_{(1,93)}=50.87 \\
p<0.001, \eta^{2}=0.35\end{array}$ \\
\hline Go > no-go & $\begin{array}{l}\text { go: }=87 \pm 12 \% \\
\text { no-go: }=73 \pm 21 \% \\
t_{(86)}=7.97, p<0.001\end{array}$ & $\begin{array}{l}\text { go: }=91 \pm 9 \% \\
\text { no-go: }=79 \pm 18 \% \\
t_{(94)}=7.68, p<0.001\end{array}$ \\
\hline Time & $\begin{array}{l}F_{(1,85)}=135.92 \\
p<0.001, \eta^{2}=0.62\end{array}$ & $\begin{array}{l}F_{(1,93)}=189.21 \\
p=<0.001, \eta^{2}=0.67\end{array}$ \\
\hline 2 nd half $>1$ st half & $\begin{array}{l}\text { 1st half: }=74 \pm 15 \% \\
\text { 2nd half: }=86 \pm 16 \% \\
t_{(86)}=11.89, p<0.001\end{array}$ & $\begin{array}{l}\text { 1st half: }=78 \pm 13 \% \\
\text { 2nd half: }=92 \pm 13 \% \\
t_{(94)}=14.68 \\
p<0.001\end{array}$ \\
\hline Action $\times$ valence & $\begin{array}{l}F_{(1,85)}=44.41 \\
p<0.001, \eta^{2}=0.34\end{array}$ & $\begin{array}{l}F_{(1,93)}=37.72 \\
p<0.001, \eta^{2}=0.29\end{array}$ \\
\hline $\begin{array}{l}\text { Go to win > go to } \\
\text { avoid losing }\end{array}$ & $\begin{array}{l}\text { gw: }=91 \pm 14 \% \\
\text { gal: }=82 \pm 14 \% \\
t_{(86)}=6.28, p<0.001\end{array}$ & $\begin{array}{l}\text { gw: }=95 \pm 12 \% \\
\text { gal: }=87 \pm 10 \% \\
t_{(94)}=5.74, p<0.001\end{array}$ \\
\hline $\begin{array}{l}\text { No-go to avoid losing } \\
>\text { no-go to win }\end{array}$ & $\begin{array}{l}\text { ngw: }=66 \pm 32 \% \\
\text { ngal: }=81 \pm 16 \% \\
t_{(86)}=4.99, p<0.001\end{array}$ & $\begin{array}{l}\text { ngw: }=73 \pm 30 \% \\
\text { ngal: }=86 \pm 11 \% \\
t_{(94)}=4.63, p<0.001\end{array}$ \\
\hline Action $\times$ time & $\begin{array}{l}F_{(1,85)}=19.09 \\
p<0.001, \eta^{2}=0.18\end{array}$ & $\begin{array}{l}F_{(1,93)}=59.77 \\
p<0.001, \eta^{2}=0.39\end{array}$ \\
\hline $\begin{array}{l}\text { 1st half(go-no-go) } \\
>2 \text { nd } \\
\text { half(go-no-go) }\end{array}$ & $\begin{array}{l}\text { 1st half: }=17 \pm 17 \% \\
\text { 2nd half: }=9 \pm 18 \% \\
t_{(86)}=4.62, p<0.001\end{array}$ & $\begin{array}{l}\text { 1st half: }=18 \pm 17 \% \\
\text { 2nd half: }=6 \pm 16 \% \\
t_{(94)}=8.46, p<0.001\end{array}$ \\
\hline $\begin{array}{l}\text { Action } \times \text { valence } \times \\
\text { time } \times \text { genotype }\end{array}$ & $\begin{array}{l}F_{(1,85)}=5.24 \\
p=0.025, \eta^{2}=0.06\end{array}$ & $\begin{array}{l}F_{(1,93)}=4.59 \\
p=0.035, \eta^{2}=0.05\end{array}$ \\
\hline $\begin{array}{l}\text { A1-(ngw(2nd-1st } \\
\text { half)) }> \\
\text { A1+(ngw(2nd-1st } \\
\text { half)) }\end{array}$ & $\begin{array}{l}\mathrm{A} 1+:=8 \pm 21 \% \\
\mathrm{~A} 1-:=22 \pm 26 \% \\
t_{(85)}=2.78, p=0.007\end{array}$ & $\begin{array}{l}\mathrm{A} 1+:=15 \pm 22 \% \\
\mathrm{~A} 1-:=25 \pm 24 \% \\
t_{(93)}=2.16, p=0.033\end{array}$ \\
\hline
\end{tabular}

Means \pm standard deviations are shown. Only effects that were significant in both cohorts are reported. ANOVA was computed with percent correct responses as dependent variable and action, valence, time and genotype as independent variables. Paired t-tests and t-tests for independent samples were performed as post-hoc tests. gw, go to win; gal, go to avoid losing; ngw, nogo to win; ngal, no-go to avoid losing. A1+; carriers of the A1 allele. A1-; A2 homozygotes.

in the separate cohorts $\left[F_{(1,179)}=9.87, p=0.002\right]$. Only in one cohort there was a statistically significant three-way interaction [action by valence by time; cohort $1: F_{(1,85)}=0.42, p=$ 0.517 ; cohort 2: $\left.F_{(1,93)}=10.98, p=0.001\right]$ and a time by genotype interaction [cohort 1: $F_{(1,85)}=3.77, p=0.055$; cohort 2 : $\left.F_{(1,93)}=6.31, p=0.014\right]$.

Statistics regarding reaction times (RTs) of the go responses are summarized in Table 4. We computed an ANOVA with valence (win/lose) as within-subject factor and genotype as betweensubject factor. Irrespective of genotype, RTs in the go to win condition were shorter than in the go to avoid losing condition [cohort 1: $F_{(1,85)}=14.06, p<0.001$; cohort $2: F_{(1,93)}=11.21$, $p=0.001]$. Regarding DRD2/ANKK1 TaqIA genotype, there was only a trendwise interaction with valence $\left[F_{(1,93)}=3.38, p=\right.$ $0.069]$ and a trend for a main effect $\left[F_{(1,93)}=3.67, p=0.058\right]$ in cohort 2, with the A1 carriers being slower in avoiding punishment as compared to the A2 homozygotes $\left[t_{(93)}=2.04, p=\right.$ 0.046]. Although this nominal effect together with the worse accuracy of the A1 carriers in the go to avoid losing condition (Figure 2) hints at a worse performance of the Al carriers in this condition, the interpretation of this result warrants caution as the effects were only apparent in cohort 2 and, moreover, participants were explicitly instructed to respond accurately, while speed was not emphasized.

To rule out that the genotype effects are not simply explained by differences in target detection performance the percentage of trials in which subjects responded incorrectly in the target detection task (i.e., left when the target was on the right side of the display or vice versa) was measured and did not differ significantly between genotype groups (Mann-Whitney $U$-test: cohort 1: Al+: $M \pm S D=1 \pm 3 \%, \mathrm{Al}-: M \pm S D=1 \pm 2 \%$, $z=-0.334, p=0.738$; cohort 2: A1+: $M \pm S D=1 \pm 3 \%, \mathrm{~A} 1-$ : $M \pm S D=0 \pm 1 \%, z=-0.428, p=0.668)$.

Because the TaqIA polymorphism is located downstream of the DRD2 gene, the observed genotype effects might putatively result from linkage disequilibrium with other DRD2 polymorphisms, including the C957T. We indeed observed an imbalanced distribution of the C957T polymorphism (rs6277) among TaqIA A1 carriers vs. A2 homozygotes numerically in the first cohort $\left(\chi^{2}=4.04, p=0.132\right)$ and significantly in the second cohort $\left(\chi^{2}=25.49, p<0.001\right)$. Moreover, the DARPP-32 polymorphism (rs907094) was unequally distributed in the second cohort only $\left(\chi^{2}=8.53, p=0.014\right)$. In order to rule out confounding effects, we included the polymorphisms as covariates in an additional ANCOVA. The same was done for COMT Val108/158 Met (rs4680), because the cohorts were stratified with respect to that polymorphism. Importantly, the four-fold action by valence by time by genotype interaction for the TaqIA polymorphism remained significant [cohort $1: F_{(1,82)}=4.63, p=0.034$, cohort 2: $\left.F_{(1,90)}=5.07, p=0.027\right]$, while there was no effect for C957T (cohort 1: $p=0.472$, cohort 2: $p=0.810$ ), DARPP-32 (cohort 1: $p=0.578$, cohort $2: p=0.148)$ or COMT Val108/158 Met polymorphism (cohort $1: p=0.161$, cohort $2: p=0.856$ ).

\section{DISCUSSION}

The goal of this study was to investigate how a genetic variant linked to striatal DA responsivity affects the action/valence interaction. To this end, two independent cohorts consisting of 87 and 95 healthy participants were genotyped for the well-characterized DRD2/ANKK1 TaqIA polymorphism (Grandy et al., 1989; Dubertret et al., 2004; Neville et al., 2004) and performed the previously described valenced go/no-go task (GuitartMasip et al., 2012b, 2013, 2014; Cavanagh et al., 2013; Chowdhury et al., 2013). Our results show differential learning performance in the carriers of the less common Al allele of the TaqIA polymorphism, which has previously been linked to lower striatal dopamine D2 receptor expression. Replicating previous results, 


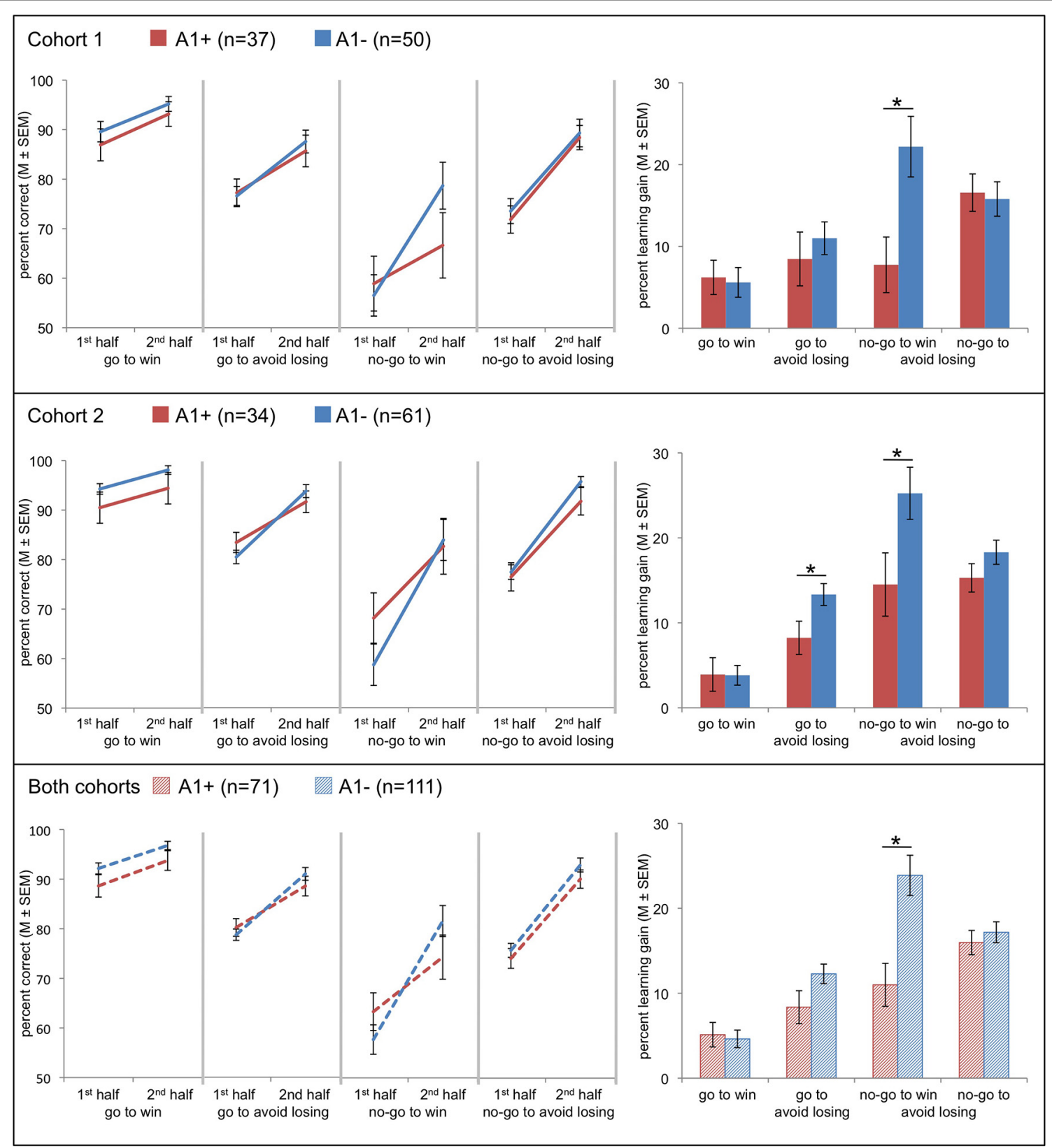

FIGURE 2 | Effects of Taq1A genotype on choice performance in two independent cohorts and in the entire sample (data of both cohorts combined). Line charts at the left show mean values of correct responses ( \pm s.e.m.) in $A 1$ carriers (red) and $A 2$ homozygotes (blue) in the first and the second half of trials for all four conditions. Bar plots at the right show the differences between mean ( \pm s.e.m.) values of correct responses of second half of trials minus first half of trials in A1 carriers (red) and A2 homozygotes (blue) for each condition. This score represents the four-fold interaction of action by valence by time by genotype. Compared to the A2 homozygotes carriers of the A1 allele showed a diminished learning to withhold an action to receive a reward Post-hoc comparisons via t-test: $* p<0.05$ participants were, irrespective of genotype, more successful in learning active choices in rewarded conditions and passive choices in punished conditions, with response inhibition to obtain a reward (no-go to win) being the condition most difficult to learn. The DRD2 TaqIA polymorphism exerted a modulatory influence on learning performance in the no-go to win condition with A1 carriers showing lower learning rates throughout the experiment.
It has to be emphasized that, despite the fact that in the present study learning curves of the two cohorts differed to some extent and initial performance of A1 carriers was not identical, we did yet observe a replicable attenuation of learning rates in A1 carriers that was specific to the no-go to win condition, and, importantly, the effect was even more pronounced when combining both datasets (using cohort as a covariate of no interest; see Figure 2). 
Table 4 | Statistics on reaction times of correct go responses.

\begin{tabular}{|c|c|c|c|}
\hline & $\mathbf{A} 1+$ & A1- & \\
\hline \multicolumn{4}{|l|}{ COHORT 1} \\
\hline Go to win & $527 \pm 128 \mathrm{~ms}$ & $535 \pm 88 \mathrm{~ms}$ & $t_{(85)}=-0.36, p=0.719$ \\
\hline Go to avoid losing & $547 \pm 129 \mathrm{~ms}$ & $564 \pm 117 \mathrm{~ms}$ & $t_{(85)}=-0.65, p=0.521$ \\
\hline \multicolumn{4}{|l|}{ COHORT 2} \\
\hline Go to win & $561 \pm 100 \mathrm{~ms}$ & $534 \pm 76 \mathrm{~ms}$ & $t_{(93)}=1.48, p=0.144$ \\
\hline Go to avoid losing & $583 \pm 107 \mathrm{~ms}$ & $540 \pm 76 \mathrm{~ms}$ & $t_{(93)}=2.04, p=0.046$ \\
\hline
\end{tabular}

Means \pm standard deviations are shown. A1+; carriers of the A1 allele. A1-;A2 homozygotes.

It is important to note that there are two potential mechanisms by which valence can disrupt the choice of appropriate actions in the current task. The first mechanism is implemented at the time of the choice and can be seen as "Pavlovian" mechanism by which the anticipation of reward or punishment promotes action or inhibition, respectively (Dayan et al., 2006; Huys et al., 2011; Guitart-Masip et al., 2012b). The second mechanism is implemented at the time of outcome and is related to the role of DA within the striatum. According to a prevalent view in reinforcement learning and decision making, DA neurons signal reward prediction errors (Montague et al., 1996; Schultz et al., 1997; Bayer and Glimcher, 2005), in the form of phasic bursts for positive prediction errors and dips below baseline firing rate for negative prediction errors (Bayer et al., 2007), resulting in corresponding peaks and dips of dopamine availability in target structures, most prominently the striatum (McClure et al., 2003; O'Doherty et al., 2003, 2004; Pessiglione et al., 2006). In the striatum, increases of DA in response to an unexpected reward reinforce the direct pathway via activation of D1 receptors and thereby facilitate the future generation of go choices under similar circumstances, while dips in DA levels in response to an unexpected punishment reinforce the indirect pathway via reduced activation of D2 receptors and thus facilitate the subsequent generation of no-go choices in comparable situations (Frank et al., 2004, 2007; Wickens et al., 2007; Hikida et al., 2010; see Figure 3).

The effects related to the TaqIA polymorphism observed in the present study apparently reflect changes in the learning process, thus likely pointing to the function of DA in the ability to flexibly learn go or no-go choices based on the outcomes produced by previous actions. Our results are in apparent contrast to the effects previously reported in the same task after administration of levodopa. In that study, boosting DA levels resulted in a decoupling between action and valence that did not reflect any changes in the rate of learning (Guitart-Masip et al., 2013). Instead, the effects observed in that study boosted the asymptote reached by the participants that received levodopa. Using computational modeling, that effect was best characterized as a decreased influence of a Pavlovian control mechanism over the instrumental control mechanisms attempting to learn the task (Guitart-Masip et al., 2013). Similarly, in older adults, structural MRI measures of substantia nigra/ventral tegmental area (SN/VTA) integrity have also been linked to improved learning and a lower action bias (Chowdhury et al., 2013). One proposed explanation for the reduced coupling between action and valence in conditions associated with increased DA availability has been a likely increase of dopaminergic activity in the prefrontal cortex where DA influences the balance between different control mechanisms (Hitchcott et al., 2007). The implication of a prefrontal mechanism decreasing the Pavlovian influences on behavior and supporting performance of the no-go to win condition in this task has been shown in fMRI (Guitart-Masip et al., 2012b) and EEG experiments (Cavanagh et al., 2013). It should be noted, though, that, in the present study, we did not observe any behavioral differences as a function of the COMT Val108/158 Met polymorphism, which has previously been linked to prefrontal dopamine availability (Meyer-Lindenberg et al., 2005).

Receptor binding studies in vitro and in vivo have shown that A1 carriers show lower striatal D2 receptor expression (Noble et al., 1991; Thompson et al., 1997; Pohjalainen et al., 1998; Jonsson et al., 1999; Ritchie and Noble, 2003). On the other hand, A1 carriers also exhibit increased striatal DA synthesis, possibly as a result of reduced autoinhibitory signaling from presynaptic D2-type autoreceptors (Laakso et al., 2005). Previous behavioral and neuroimaging studies have in fact yielded results that would be best explained by parallel reduction of striatal postsynaptic D2 receptors and increased presynaptic dopaminergic activity in A1 carriers, with the latter also resulting in increased DA availability both in the striatum and in extrastriatal regions (Kirsch et al., 2006; Stelzel et al., 2010; Richter et al., 2013). According to those observations, A1 carriers would be assumed to show a less pronounced decrease of dopaminergic signaling after negative prediction errors in the indirect pathway and a shift to a more action-oriented behavioral pattern mediated by the direct pathway (Figure 3). Such a pattern bears some resemblance to the concept of behavioral impulsivity (Tomie et al., 1998; Flagel et al., 2010, 2011), and it is noteworthy in this context that the A1 allele has been linked to risk for impulsivity-related psychiatric disorders, most prominently alcohol dependence (Noble et al., 1991; Comings et al., 1996; Noble, 2003; Eisenberg et al., 2007; Wang et al., 2013). However, this does not explain, why A1 carriers exhibit a relatively specific performance disadvantage in the no-go to win, but not in the no-go to avoid losing condition. One possible reason would be that a punishment instead of a neutral feedback in the no-go to avoid losing condition might lead to a higher prediction error as compared to a neutral feedback instead of a reward in the no-go to win condition. Another reason might be that, for example, serotonin plays a specific role in punishmentrelated behavior (Daw et al., 2002; Boureau and Dayan, 2011; Cools et al., 2011; Guitart-Masip et al., 2012b, 2013; Den Ouden et al., 2013) and thus further modulates the performance in the no-go to avoid losing condition.

The investigation of modulators of stereotyped hard-wired behavioral responses is of interest to clinicians as it may help to develop novel treatment approaches for neurological or psychiatric disorders. The TaqIA polymorphism is one of the most extensively studied genetic variations in neuropsychiatric disorders with presumed dopaminergic dysfunction, and studies have pointed to a potential pleiotropic effect with A1 allele carriers showing an increased risk for addiction, but a lower risk for schizophrenia (e.g., Comings et al., 1996; Noble, 2003; Dubertret et al., 2004; Wang et al., 2013; Zhang et al., 2014). Moreover, 


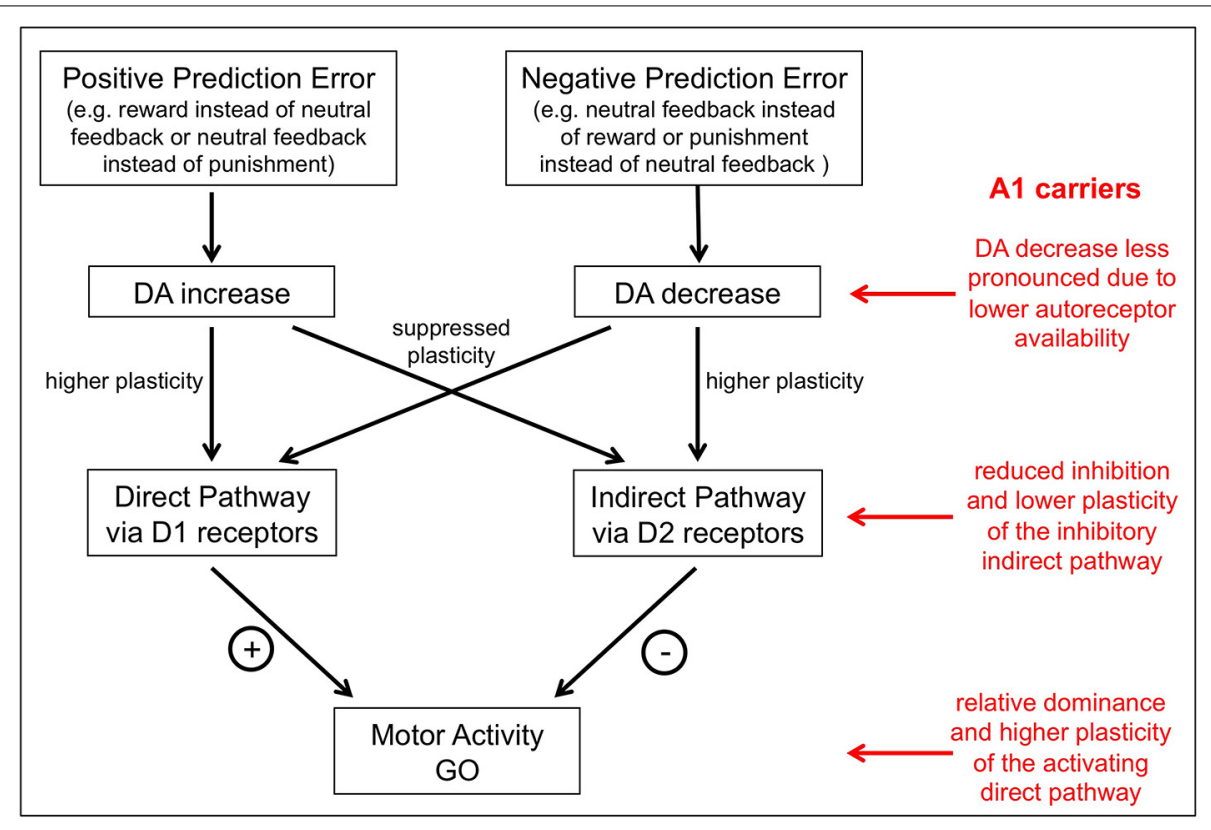

FIGURE 3 | A model of the putative influence of the TaqIA polymorphism on action-valence interaction. DA neurons signal reward prediction errors in the form of phasic bursts for positive prediction errors and dips below baseline firing rate for negative prediction errors. Increases of DA in response to an unexpected reward reinforce the direct pathway via activation of D1 receptors and thereby facilitate the future generation of go choices under similar circumstances, while dips in DA levels in response to an unexpected punishment reinforce the indirect pathway via reduced activation of D2 receptors and thus facilitate the subsequent generation of no-go choices in comparable situations. A1 carriers have less D2 receptors and thus would be assumed to have less limitation of dopaminergic signaling after negative prediction errors in the indirect pathway and a shift to a more action-oriented behavioral pattern mediated by the direct pathway. studies in healthy humans have suggested a role of the TaqIA A1 variant in approach-related personality traits (Noble et al., 1998; Reuter et al., 2006; Lee et al., 2007; Smillie et al., 2010) and on motivated interference processing (Richter et al., 2013). The relation between the single nucleotide polymorphism (SNP) and instrumental learning has also been investigated. Previous studies have shown an impairment of the carriers of the A1 allele in no-go learning to avoid behaviors that yield negative outcomes (Klein et al., 2007; Frank and Hutchison, 2009; Jocham et al., 2009). However, those studies have only used conditions in which participants had to approach a reward or avoid a punishment. Since the interaction between action and valence has a pivotal influence on instrumental learning (Guitart-Masip et al., 2012b), such studies could not provide information on possible action by valence interactions, and the use of the valenced go/no-go-learning task with orthogonalized action and valence enables a more precise investigation of the contribution of the dopaminergic system in behavioral adaptation.

The TaqIA polymorphism, initially identified to be located on the DRD2 gene on human chromosome 11q22-23 (Grandy et al., 1989 ), is located $10 \mathrm{~kb}$ downstream of the DRD2 termination codon on 11q23.1, within coding region of the adjacent ankyrin repeat and kinase domain containing 1 (ANKK1) gene (Dubertret et al., 2004; Neville et al., 2004). Because the DRD2 and ANKK1 genes are closely linked (Neville et al., 2004; Ponce et al., 2009), it has been proposed that genetic variations in linkage disequilibrium (LD) with the SNP might explain the observed relationship between the TaqIA and alterations of human dopaminergic neurotransmission. The SNP is indeed in LD with several polymorphisms on the DRD2 gene (Duan et al., 2003; Ritchie and Noble, 2003; Fossella et al., 2006) and one of them is the C957T polymorphism (rs6277) for which also modulations on instrumental learning have been observed (Frank et al., 2007, 2009; Frank and Hutchison, 2009). However, its influence on dopaminergic neurotransmission is not clear since in vivo and in vitro data are in conflict (Duan et al., 2003; Hirvonen et al., 2004; see also erratum by Hirvonen et al., 2004, 2009a,b) and no association was found between C957T and DA synthesis capacity in vivo (Laakso et al., 2005) and C957T and D2 receptor mRNA expression in post mortem brain tissue (Zhang et al., 2007). When controlling for a potential influence of this SNP in our analysis, the effect of TaqIA genotype was still significant. We cannot rule out, though, that another variant in the DRD2 gene-or perhaps in the ANKK1 gene-linked to TaqIA might be responsible for the observed genotype-related differences in learning rate.

In order to control for genetic influences of another genetic variant known to affect prefrontal DA levels and thereby cortical D1 receptor stimulation (Gogos et al., 1998; Matsumoto et al., 2003; Meyer-Lindenberg et al., 2005; Slifstein et al., 2008) we selected our participants to have comparable distributions of the COMT Val108/158 Met genotype. Importantly, the allelic distribution of COMT Val108/158 Met alleles did not differ significantly between TaqIA A1 carriers and A2 homozygotes.

It must nevertheless be kept in mind that genetic variations within the dopaminergic system do not exert their effects in isolation. Frank et al. (2007), for example, observed multiple 
roles for DA in reinforcement learning when investigating effects of the COMT Val108/158 Met, the DARPP-32, and the DRD2 C957T polymorphism on reward-based probabilistic learning. Even though we controlled for these polymorphisms in our experiment, we cannot completely rule out gene-gene interactions. Our moderately large sample sizes allowed us to examine effects of single genetic variants on behavioral outcomes, but the systematic analysis of gene-gene interactions would require substantially larger cohorts. In addition to the likely polygenic contribution of variants in the dopaminergic system to action by valence interaction, also other neuromodulatory transmitters must be considered in future studies.

\section{CONCLUSION}

Our findings provide further evidence for a potential genetic basis of individual differences in probabilistic learning and, more specifically, suggest that genetically mediated differences in dopaminergic neuromodulation not only affect learning per $s e$, but also can specifically affect behavioral phenomena like a Pavlovian action bias when a reward is expected. With respect to future research directed at individual differences in learning, our findings should thereby caution researchers to take into account the non-orthogonal nature of action by valence interactions.

\section{ACKNOWLEDGMENTS}

The authors would like to thank Iris Mann for help with testing and Maria Michelmann for help with genotyping. This project was supported by the Deutsche Forschungsgemeinschaft (SFB 779, TP A07 and A08) and the Leibniz Graduate School (PhD stipend to Adriana Barman, Master stipend to Marieke Klein). The authors have no conflicts of interest or involvement, financial or otherwise, to report.

\section{REFERENCES}

Bayer, H. M., and Glimcher, P. W. (2005). Midbrain dopamine neurons encode a quantitative reward prediction error signal. Neuron 47, 129-141. doi: 10.1016/j.neuron.2005.05.020

Bayer, H. M., Lau, B., and Glimcher, P. W. (2007). Statistics of midbrain dopamine neuron spike trains in the awake primate. J. Neurophysiol. 98, 1428-1439. doi: 10.1152/jn.01140.2006

Beierholm, U., Guitart-Masip, M., Economides, M., Chowdhury, R., Duzel, E., Dolan, R., et al. (2013). Dopamine modulates reward-related vigor. Neuropsychopharmacology 38, 1495-1503. doi: 10.1038/npp.2013.48

Berridge, K. C., and Robinson, T. E. (1998). What is the role of dopamine in reward: hedonic impact, reward learning, or incentive salience? Brain Res. Brain Res. Rev. 28, 309-369.

Boureau, Y. L., and Dayan, P. (2011). Opponency revisited: competition and cooperation between dopamine and serotonin. Neuropsychopharmacology 36, 74-97. doi: $10.1038 /$ npp. 2010.151

Breland, K., and Breland, M. (1961). The misbehavior of organisms. Am. Psychol. 16, 681-684. doi: 10.1037/h0040090

Cavanagh, J. F., Eisenberg, I., Guitart-Masip, M., Huys, Q., and Frank, M. J. (2013). Frontal theta overrides pavlovian learning biases. J. Neurosci. 33, 8541-8548. doi: 10.1523/JNEUROSCI.5754-12.2013

Chowdhury, R., Guitart-Masip, M., Lambert, C., Dolan, R. J., and Duzel, E. (2013). Structural integrity of the substantia nigra and subthalamic nucleus predicts flexibility of instrumental learning in older-age individuals. Neurobiol. Aging 34, 2261-2270. doi: 10.1016/j.neurobiolaging.2013.03.030

Comings, D. E., and Blum, K. (2000). Reward deficiency syndrome: genetic aspects of behavioral disorders. Prog. Brain Res. 126, 325-341. doi: 10.1016/S00796123(00)26022-6
Comings, D. E., Rosenthal, R. J., Lesieur, H. R., Rugle, L. J., Muhleman, D., Chiu, C., et al. (1996). A study of the dopamine D2 receptor gene in pathological gambling. Pharmacogenetics 6, 223-234. doi: 10.1097/00008571-199606000-00004

Cools, R., Nakamura, K., and Daw, N. D. (2011). Serotonin and dopamine: unifying affective, activational, and decision functions. Neuropsychopharmacology 36, 98-113. doi: 10.1038/npp.2010.121

Daw, N. D., and Doya, K. (2006). The computational neurobiology of learning and reward. Curr. Opin. Neurobiol. 16, 199-204. doi: 10.1016/j.conb.2006.03.006

Daw, N. D., Kakade, S., and Dayan, P. (2002). Opponent interactions between serotonin and dopamine. Neural Netw. 15, 603-616. doi: 10.1016/S08936080(02)00052-7

Dayan, P., Niv, Y., Seymour, B., and Daw, N. D. (2006). The misbehavior of value and the discipline of the will. Neural Netw. 19, 1153-1160. doi: 10.1016/j.neunet.2006.03.002

Den Ouden, H. E., Daw, N. D., Fernandez, G., Elshout, J. A., Rijpkema, M., Hoogman, M., et al. (2013). Dissociable effects of dopamine and serotonin on reversal learning. Neuron 80, 1090-1100. doi: 10.1016/j.neuron.2013.08.030

Dickinson, A., and Balleine, B. (2002). "The role of learning in the operation of motivational systems," in Steven's Handbook of Experimental Psychology: Learning, Motivation and Emotion, 3rd Edn., Vol. 3, ed C. R. Gallistel (New York, NY: John Wiley \& Sons), 497-534.

Duan, J., Wainwright, M. S., Comeron, J. M., Saitou, N., Sanders, A. R., Gelernter, J., et al. (2003). Synonymous mutations in the human dopamine receptor D2 (DRD2) affect mRNA stability and synthesis of the receptor. Hum. Mol. Genet. 12, 205-216. doi: 10.1093/hmg/ddg055

Dubertret, C., Gouya, L., Hanoun, N., Deybach, J. C., Ades, J., Hamon, M., et al. (2004). The $3^{\prime}$ region of the DRD2 gene is involved in genetic susceptibility to schizophrenia. Schizophr. Res. 67, 75-85. doi: 10.1016/S0920-9964(03) 00220-2

Eisenberg, D. T., Mackillop, J., Modi, M., Beauchemin, J., Dang, D., Lisman, S. A., et al. (2007). Examining impulsivity as an endophenotype using a behavioral approach: a DRD2 TaqI A and DRD4 48-bp VNTR association study. Behav. Brain Funct. 3:2.

Flagel, S. B., Clark, J. J., Robinson, T. E., Mayo, L., Czuj, A., Willuhn, I., et al. (2011). A selective role for dopamine in stimulus-reward learning. Nature 469, 53-57. doi: 10.1038/nature09588

Flagel, S. B., Robinson, T. E., Clark, J. J., Clinton, S. M., Watson, S. J., Seeman, P., et al. (2010). An animal model of genetic vulnerability to behavioral disinhibition and responsiveness to reward-related cues: implications for addiction. Neuropsychopharmacology 35, 388-400. doi: 10.1038/npp. 2009.142

Fossella, J., Green, A. E., and Fan, J. (2006). Evaluation of a structural polymorphism in the ankyrin repeat and kinase domain containing 1 (ANKK1) gene and the activation of executive attention networks. Cogn. Affect. Behav. Neurosci. 6, 71-78. doi: 10.3758/CABN.6.1.71

Frank, M. J., Doll, B. B., Oas-Terpstra, J., and Moreno, F. (2009). Prefrontal and striatal dopaminergic genes predict individual differences in exploration and exploitation. Nat. Neurosci. 12, 1062-1068. doi: 10.1038/nn.2342

Frank, M. J., and Hutchison, K. (2009). Genetic contributions to avoidance-based decisions: striatal D2 receptor polymorphisms. Neuroscience 164, 131-140. doi: 10.1016/j.neuroscience.2009.04.048

Frank, M. J., Moustafa, A. A., Haughey, H. M., Curran, T., and Hutchison, K. E. (2007). Genetic triple dissociation reveals multiple roles for dopamine in reinforcement learning. Proc. Natl. Acad. Sci. U.S.A. 104, 16311-16316. doi: 10.1073/pnas.0706111104

Frank, M. J., Seeberger, L. C., and O'Reilly, R. C. (2004). By carrot or by stick: cognitive reinforcement learning in parkinsonism. Science 306, 1940-1943. doi: 10.1126/science.1102941

Gogos, J. A., Morgan, M., Luine, V., Santha, M., Ogawa, S., Pfaff, D., et al. (1998). Catechol-O-methyltransferase-deficient mice exhibit sexually dimorphic changes in catecholamine levels and behavior. Proc. Natl. Acad. Sci. U.S.A. 95, 9991-9996. doi: 10.1073/pnas.95.17.9991

Grandy, D. K., Litt, M., Allen, L., Bunzow, J. R., Marchionni, M., Makam, H., et al. (1989). The human dopamine D2 receptor gene is located on chromosome 11 at q22-q23 and identifies a TaqI RFLP. Am. J. Hum. Genet. 45, 778-785.

Gray, J. A., and McNaughton, M. (2000). The Neuropsychology of Anxiety: An Inquiry into the Function of the Septohippocampal System, 2nd Edn. Oxford: Oxford University Press 
Guitart-Masip, M., Chowdhury, R., Sharot, T., Dayan, P., Duzel, E., and Dolan, R. J. (2012a). Action controls dopaminergic enhancement of reward representations. Proc. Natl. Acad. Sci. U.S.A. 109, 7511-7516. doi: 10.1073/pnas.1202229109

Guitart-Masip, M., Duzel, E., Dolan, R., and Dayan, P. (2014). Action versus valence in decision making. Trends Cogn. Sci. 18, 194-202. doi: 10.1016/j.tics.2014.01.003

Guitart-Masip, M., Economides, M., Huys, Q. J., Frank, M. J., Chowdhury, R., Duzel, E., et al. (2013). Differential, but not opponent, effects of L DOPA and citalopram on action learning with reward and punishment. Psychopharmacology (Berl) 231, 955-966. doi: 10.1007/s00213-013-3313-4

Guitart-Masip, M., Huys, Q. J., Fuentemilla, L., Dayan, P., Duzel, E., and Dolan, R. J. (2012b). Go and no-go learning in reward and punishment: interactions between affect and effect. Neuroimage 62, 154-166. doi: 10.1016/j.neuroimage.2012.04.024

Hikida, T., Kimura, K., Wada, N., Funabiki, K., and Nakanishi, S. (2010). Distinct roles of synaptic transmission in direct and indirect striatal pathways to reward and aversive behavior. Neuron 66, 896-907. doi: 10.1016/j.neuron.2010.05.011

Hirvonen, M., Laakso, A., Nagren, K., Rinne, J. O., Pohjalainen, T., and Hietala, J. (2004). C957T polymorphism of the dopamine D2 receptor (DRD2) gene affects striatal DRD2 availability in vivo. Mol. Psychiatry 9, 1060-1061. doi: 10.1038/sj.mp.4001561

Hirvonen, M. M., Laakso, A., Nagren, K., Rinne, J. O., Pohjalainen, T., and Hietala, J. (2009a). C957T polymorphism of dopamine D2 receptor gene affects striatal DRD2 in vivo availability by changing the receptor affinity. Synapse 63, 907-912. doi: 10.1002/syn.20672

Hirvonen, M. M., Lumme, V., Hirvonen, J., Pesonen, U., Nagren, K., Vahlberg, T., et al. (2009b). C957T polymorphism of the human dopamine D2 receptor gene predicts extrastriatal dopamine receptor availability in vivo. Prog. Neuropsychopharmacol. Biol. Psychiatry 33, 630-636. doi: 10.1016/j.pnpbp.2009.02.021

Hitchcott, P. K., Quinn, J. J., and Taylor, J. R. (2007). Bidirectional modulation of goal-directed actions by prefrontal cortical dopamine. Cereb. Cortex 17, 2820-2827. doi: 10.1093/cercor/bhm010

Huys, Q. J., Cools, R., Golzer, M., Friedel, E., Heinz, A., Dolan, R. J., et al. (2011). Disentangling the roles of approach, activation and valence in instrumental and pavlovian responding. PLoS Comput. Biol. 7:e1002028. doi: 10.1371/journal.pcbi. 1002028

Jocham, G., Klein, T. A., Neumann, J., Von Cramon, D. Y., Reuter, M., and Ullsperger, M. (2009). Dopamine DRD2 polymorphism alters reversal learning and associated neural activity. J. Neurosci. 29, 3695-3704. doi: 10.1523/JNEUROSCI.5195-08.2009

Jonsson, E. G., Nothen, M. M., Grunhage, F., Farde, L., Nakashima, Y., Propping, P., et al. (1999). Polymorphisms in the dopamine D2 receptor gene and their relationships to striatal dopamine receptor density of healthy volunteers. Mol. Psychiatry 4, 290-296. doi: 10.1038/sj.mp.4000532

Kirsch, P., Reuter, M., Mier, D., Lonsdorf, T., Stark, R., Gallhofer, B., et al. (2006). Imaging gene-substance interactions: the effect of the DRD2 TaqIA polymorphism and the dopamine agonist bromocriptine on the brain activation during the anticipation of reward. Neurosci. Lett. 405, 196-201. doi: 10.1016/j.neulet.2006.07.030

Klein, T. A., Neumann, J., Reuter, M., Hennig, J., Von Cramon, D. Y., and Ullsperger, M. (2007). Genetically determined differences in learning from errors. Science 318, 1642-1645. doi: 10.1126/science.1145044

Laakso, A., Pohjalainen, T., Bergman, J., Kajander, J., Haaparanta, M., Solin, O., et al. (2005). The A1 allele of the human D2 dopamine receptor gene is associated with increased activity of striatal L-amino acid decarboxylase in healthy subjects. Pharmacogenet. Genomics 15, 387-391. doi: 10.1097/01213011-200506000-00003

Laruelle, M., Gelernter, J., and Innis, R. B. (1998). D2 receptors binding potential is not affected by Taq1 polymorphism at the D2 receptor gene. Mol. Psychiatry 3, 261-265. doi: 10.1038/sj.mp.4000343

Lee, S. H., Ham, B. J., Cho, Y. H., Lee, S. M., and Shim, S. H. (2007). Association study of dopamine receptor D2 TaqI A polymorphism and reward-related personality traits in healthy Korean young females. Neuropsychobiology 56, 146-151. doi: 10.1159/000115781

Lerman, C. E., Schnoll, R. A., and Munafo, M. R. (2007). Genetics and smoking cessation improving outcomes in smokers at risk. Am. J. Prev. Med. 33, S398-S405. doi: 10.1016/j.amepre.2007.09.006
Matsumoto, M., Weickert, C. S., Akil, M., Lipska, B. K., Hyde, T. M., Herman, M. M., et al. (2003). Catechol O-methyltransferase mRNA expression in human and rat brain: evidence for a role in cortical neuronal function. Neuroscience 116, 127-137. doi: 10.1016/S0306-4522(02)00556-0

McClure, S. M., Berns, G. S., and Montague, P. R. (2003). Temporal prediction errors in a passive learning task activate human striatum. Neuron 38, 339-346. doi: 10.1016/S0896-6273(03)00154-5

Meyer-Lindenberg, A., Kohn, P. D., Kolachana, B., Kippenhan, S., McInerney-Leo, A., Nussbaum, R., et al. (2005). Midbrain dopamine and prefrontal function in humans: interaction and modulation by COMT genotype. Nat. Neurosci. 8 , 594-596. doi: 10.1038/nn1438

Montague, P. R., Dayan, P., and Sejnowski, T. J. (1996). A framework for mesencephalic dopamine systems based on predictive Hebbian learning. J. Neurosci. 16, 1936-1947.

Neville, M. J., Johnstone, E. C., and Walton, R. T. (2004). Identification and characterization of ANKK1: a novel kinase gene closely linked to DRD2 on chromosome band 11q23.1. Hum. Mutat. 23, 540-545. doi: 10.1002/humu.20039

Niv, Y., Daw, N. D., Joel, D., and Dayan, P. (2007). Tonic dopamine: opportunity costs and the control of response vigor. Psychopharmacology (Berl) 191, 507-520. doi: 10.1007/s00213-006-0502-4

Noble, E. P. (2003). D2 dopamine receptor gene in psychiatric and neurologic disorders and its phenotypes. Am. J. Med. Genet. B Neuropsychiatr. Genet. 116B, 103-125. doi: 10.1002/ajmg.b.10005

Noble, E. P., Blum, K., Ritchie, T., Montgomery, A., and Sheridan, P. J. (1991). Allelic association of the D2 dopamine receptor gene with receptorbinding characteristics in alcoholism. Arch. Gen. Psychiatry 48, 648-654. doi: 10.1001/archpsyc. 1991.01810310066012

Noble, E. P., Ozkaragoz, T. Z., Ritchie, T. L., Zhang, X., Belin, T. R., and Sparkes, R. S. (1998). D2 and D4 dopamine receptor polymorphisms and personality. Am. J. Med. Genet. 81, 257-267.

O’Doherty, J., Dayan, P., Schultz, J., Deichmann, R., Friston, K., and Dolan, R. J. (2004). Dissociable roles of ventral and dorsal striatum in instrumental conditioning. Science 304, 452-454. doi: 10.1126/science.1094285

O’Doherty, J. P., Dayan, P., Friston, K., Critchley, H., and Dolan, R. J. (2003). Temporal difference models and reward-related learning in the human brain. Neuron 38, 329-337. doi: 10.1016/S0896-6273(03)00169-7

Palmiter, R. D. (2008). Dopamine signaling in the dorsal striatum is essential for motivated behaviors: lessons from dopamine-deficient mice. Ann. N.Y. Acad. Sci. 1129, 35-46. doi: 10.1196/annals.1417.003

Pessiglione, M., Seymour, B., Flandin, G., Dolan, R. J., and Frith, C. D. (2006). Dopamine-dependent prediction errors underpin reward-seeking behaviour in humans. Nature 442, 1042-1045. doi: 10.1038/nature05051

Pohjalainen, T., Rinne, J. O., Nagren, K., Lehikoinen, P., Anttila, K., Syvalahti, E. K., et al. (1998). The A1 allele of the human D2 dopamine receptor gene predicts low D2 receptor availability in healthy volunteers. Mol. Psychiatry 3, 256-260. doi: 10.1038/sj.mp.4000350

Ponce, G., Perez-Gonzalez, R., Aragues, M., Palomo, T., Rodriguez-Jimenez, R., Jimenez-Arriero, M. A., et al. (2009). The ANKK1 kinase gene and psychiatric disorders. Neurotox. Res. 16, 50-59. doi: 10.1007/s12640-009-9046-9

Reuter, M., Schmitz, A., Corr, P., and Hennig, J. (2006). Molecular genetics support Gray's personality theory: the interaction of COMT and DRD2 polymorphisms predicts the behavioural approach system. Int. J. Neuropsychopharmacol. 9, 155-166. doi: 10.1017/S1461145705005419

Richter, A., Richter, S., Barman, A., Soch, J., Klein, M., Assmann, A., et al. (2013). Motivational salience and genetic variability of dopamine D2 receptor expression interact in the modulation of interference processing. Front. Hum. Neurosci. 7:250. doi: 10.3389/fnhum.2013.00250

Ritchie, T., and Noble, E. P. (2003). Association of seven polymorphisms of the D2 dopamine receptor gene with brain receptor-binding characteristics. Neurochem. Res. 28, 73-82. doi: 10.1023/A:1021648128758

Salamone, J. D., Correa, M., Farrar, A., and Mingote, S. M. (2007). Effort-related functions of nucleus accumbens dopamine and associated forebrain circuits. Psychopharmacology (Berl) 191, 461-482. doi: 10.1007/s00213-006-0668-9

Salamone, J. D., Correa, M., Mingote, S. M., and Weber, S. M. (2005). Beyond the reward hypothesis: alternative functions of nucleus accumbens dopamine. Curr. Opin. Pharmacol. 5, 34-41. doi: 10.1016/j.coph.2004.09.004

Schott, B. H., Minuzzi, L., Krebs, R. M., Elmenhorst, D., Lang, M., Winz, O. H., et al. (2008). Mesolimbic functional magnetic resonance imaging activations during 
reward anticipation correlate with reward-related ventral striatal dopamine release. J. Neurosci. 28, 14311-14319. doi: 10.1523/JNEUROSCI.2058-08.2008

Schott, B. H., Seidenbecher, C. I., Fenker, D. B., Lauer, C. J., Bunzeck, N., Bernstein, H. G., et al. (2006). The dopaminergic midbrain participates in human episodic memory formation: evidence from genetic imaging. J. Neurosci. 26, 1407-1417. doi: 10.1523/JNEUROSCI.3463-05.2006

Schultz, W., Dayan, P., and Montague, P. R. (1997). A neural substrate of prediction and reward. Science 275, 1593-1599. doi: 10.1126/science.275.5306.1593

Slifstein, M., Kolachana, B., Simpson, E. H., Tabares, P., Cheng, B., Duvall, M., et al. (2008). COMT genotype predicts cortical-limbic D1 receptor availability measured with [11C]NNC112 and PET. Mol. Psychiatry 13, 821-827. doi: 10.1038/mp.2008.19

Smillie, L. D., Cooper, A. J., Proitsi, P., Powell, J. F., and Pickering, A. D. (2010). Variation in DRD2 dopamine gene predicts Extraverted personality. Neurosci. Lett. 468, 234-237. doi: 10.1016/j.neulet.2009.10.095

Stelzel, C., Basten, U., Montag, C., Reuter, M., and Fiebach, C. J. (2010). Frontostriatal involvement in task switching depends on genetic differences in d2 receptor density. J. Neurosci. 30, 14205-14212. doi: 10.1523/JNEUROSCI.1062-10.2010

Thompson, J., Thomas, N., Singleton, A., Piggott, M., Lloyd, S., Perry, E. K., et al. (1997). D2 dopamine receptor gene (DRD2) Taq1 A polymorphism: reduced dopamine $\mathrm{D} 2$ receptor binding in the human striatum associated with the A1 allele. Pharmacogenetics 7, 479-484. doi: 10.1097/00008571-19971200000006

Tomie, A., Aguado, A. S., Pohorecky, L. A., and Benjamin, D. (1998). Ethanol induces impulsive-like responding in a delay-of-reward operant choice procedure: impulsivity predicts autoshaping. Psychopharmacology (Berl) 139, 376-382. doi: $10.1007 / \mathrm{s} 002130050728$

Ungerstedt, U. (1971). Adipsia and aphagia after 6-hydroxydopamine induced degeneration of the nigro-striatal dopamine system. Acta Physiol. Scand. Suppl. 367, 95-122. doi: 10.1111/j.1365-201X.1971.tb11001.x

Verde, Z., Santiago, C., Rodriguez Gonzalez-Moro, J. M., De Lucas Ramos, P., Lopez Martin, S., Bandres, F., et al. (2011). 'Smoking genes': a genetic association study. PLoS ONE 6:e26668. doi: 10.1371/journal.pone.0026668

Wang, F., Simen, A., Arias, A., Lu, Q. W., and Zhang, H. (2013). A large-scale metaanalysis of the association between the ANKK1/DRD2 Taq1A polymorphism and alcohol dependence. Hum. Genet. 132, 347-358. doi: 10.1007/s00439-0121251-6

Wickens, J. R., Budd, C. S., Hyland, B. I., and Arbuthnott, G. W. (2007). Striatal contributions to reward and decision making: making sense of regional variations in a reiterated processing matrix. Ann. N.Y. Acad. Sci. 1104, 192-212. doi: 10.1196/annals.1390.016

Wimber, M., Schott, B. H., Wendler, F., Seidenbecher, C. I., Behnisch, G., Macharadze, T., et al. (2011). Prefrontal dopamine and the dynamic control of human long-term memory. Transl. Psychiatry 1:e15. doi: 10.1038/tp. 2011.15

Zhang, L., Hu, L., Li, X., Zhang, J., and Chen, B. (2014). The DRD2 rs1800497 polymorphism increase the risk of mood disorder: evidence from an update meta-analysis. J. Affect. Disord. 158, 71-77. doi: 10.1016/j.jad.2014.01.015

Zhang, Y., Bertolino, A., Fazio, L., Blasi, G., Rampino, A., Romano, R., et al. (2007). Polymorphisms in human dopamine D2 receptor gene affect gene expression, splicing, and neuronal activity during working memory. Proc. Natl. Acad. Sci. U.S.A. 104, 20552-20557. doi: 10.1073/pnas.0707106104

Conflict of Interest Statement: The authors declare that the research was conducted in the absence of any commercial or financial relationships that could be construed as a potential conflict of interest.

Received: 25 June 2014; accepted: 18 July 2014; published online: 06 August 2014. Citation: Richter A, Guitart-Masip M, Barman A, Libeau C, Behnisch G, Czerney S, Schanze D, Assmann A, Klein M, Düzel E, Zenker M, Seidenbecher CI and Schott BH (2014) Valenced action/inhibition learning in humans is modulated by a genetic variant linked to dopamine D2 receptor expression. Front. Syst. Neurosci. 8:140. doi: 10.3389/fnsys.2014.00140

This article was submitted to the journal Frontiers in Systems Neuroscience. Copyright (C) 2014 Richter, Guitart-Masip, Barman, Libeau, Behnisch, Czerney, Schanze, Assmann, Klein, Düzel, Zenker, Seidenbecher and Schott. This is an openaccess article distributed under the terms of the Creative Commons Attribution License (CC BY). The use, distribution or reproduction in other forums is permitted, provided the original author(s) or licensor are credited and that the original publication in this journal is cited, in accordance with accepted academic practice. No use, distribution or reproduction is permitted which does not comply with these terms. 